Thorax, 1977, 32, 697-699

\title{
Oesophageal reconstruction for complete stenosis due to dystrophic epidermolysis bullosa
}

\author{
SIAVOUSH SEHHAT AND SEIED AHMAD AMIRIE \\ From The Department of Surgery, Esfahan Medical School, Esfahan, Iran
}

Sehhat, S. and Amirie, S. A. (1977). Thorax, 32, 697-699. Oesophageal reconstruction for complete stenosis due to dystrophic epidermolysis bullosa. Oesophageal involvement in epidermolysis bullosa is discussed. Two patients, a brother and sister, with stricture of the oesophagus due to the disease are described. The strictures were treated by resection and end-to-end oesophageal anastomosis and the patients have been relieved of dysphagia for the subsequent three years.

Epidermolysis bullosa is a rare chronic hereditary disorder involving the skin and mucous membranes characterised by the development of bullae, which may appear spontaneously or as a result of trauma.

Two main types of epidermolysis bullosa are recognised (Newcomer et al., 1956; Schnyder and Eichhoff, 1964; Gedde-Dahl, 1971). The simple type occurs mainly in infants and rarely affects the mucosae. In the second type, hyperplastic and dystrophic epidermolysis bullosa, the mucosae are involved in $23 \%$ of patients. In addition, a third less common and more serious type has been described, namely, polydysplastic epidermolysis bullosa.

Involvement of the oesophagus during the course of epidermolysis bullosa is uncommon. Nix and Christianson (1965) collected 24 cases of oesophageal involvement from the world literature between 1899 and 1962 and added two new cases, both of whom had severe stenosis and died of starvation. Bergenholtz et al. (1963) reviewed 18 patients, 13 male and 5 female, with an age range from birth to the fourth decade. They noted that the oesophageal involvement was usually in the proximal or distal thirds of the oesophagus. In four patients there was involvement of the whole oesophagus. Dupree et al. (1969) reported oesophageal stenoses ranging from 2 to $6 \mathrm{~cm}$ in length.

The purpose of this report is to describe a simple approach to the problem of oesophageal stenosis complicating epidermolysis bullosa.

\section{Case reports}

CASE 1

The patient was a 37 -year-old white man. There was a history of consanguinity in his parents. He was one of a family of five. The father had suffered from bullous lesions, affecting particularly the neck and waist, throughout most of his life. One sister died at the age of 40 days with severe generalised bullous lesions. A brother with similar bullous disease died at the age of 18 months. Another brother did not suffer from the disease. A sister aged 35, who suffered from epidermolysis bullosa, is the second case reported in this paper.

The patient (case 1) had a history of bullous lesions, some of them haemorrhagic, in different parts of the body since birth. Several finger nails were reddish in colour and diminished in size. The patient had scars all over the body as a result of exfoliation of the skin with subsequent healing. The teeth were carious and of uneven growth. The diagnosis of epidermolysis bullosa was made from the clinical appearances and the family history.

At the age of 25 the patient noticed difficulty in chewing and swallowing solid food because of haemorrhagic bullous lesions in the mouth. A barium swallow showed a narrowed segment in the upper oesophagus. The lesions gradually became worse, and at the age of 28 the patient was unable to open his mouth completely and had severe dysphagia. An oesophagogram showed more severe stenosis. One year later he was able to swallow liquids and soft food only.

Eight years later the oesophagus was completely obstructed and the patient was unable to swallow saliva. A gastrostomy was done. One year later, at the time of his admission to hospital, a barium swallow showed an almost complete stenosis just below the pharyngo-oesophageal junction (Fig. 1). He was operated on through an oblique incision on 


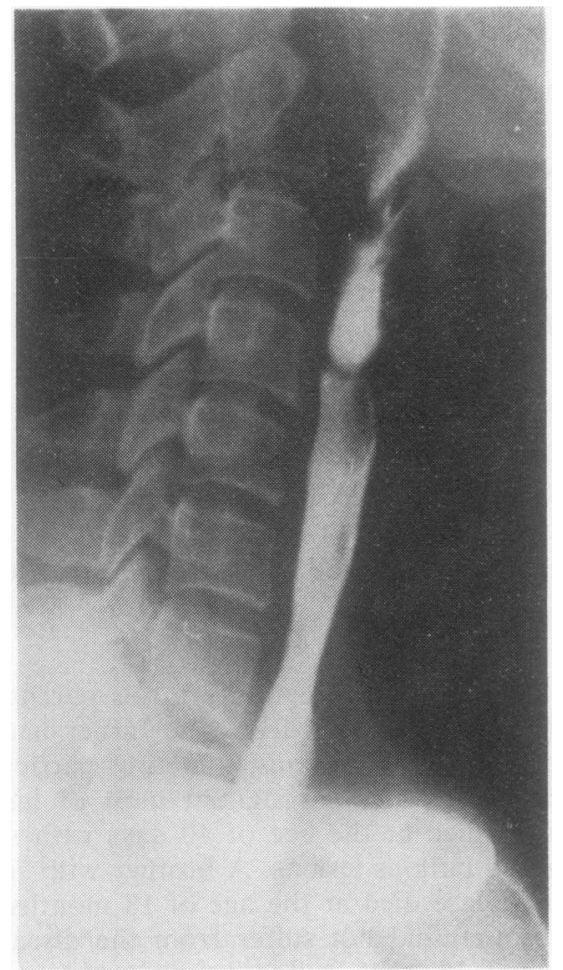

Fig. 1 Case 1. Preoperative barium swallow showing severe oesophageal obstruction.

the left side of the neck. A stenotic segment, $1.5 \mathrm{~cm}$ in length, was resected and an end-to-end anastomosis carried out. He started to swallow immediately after the operation and a barium swallow showed a lumen estimated at $75 \%$ of normal (Fig. 2). The patient was able to swallow normal food and the gastrostomy tube was removed four months later. Three years later the patient has no difficulty in swallowing.

\section{CASE 2}

A 35-year-old woman, the sister of case 1, was admitted to hospital because of severe dysphagia. There was a history of bullous lesions which began to appear shortly after birth. She had noticed increasing dysphagia over a period of eight years. On occasions she had aphagia lasting for a week at a time. The patient was thin. She was unable to open her mouth completely or to protrude her tongue. There were scars on several parts of the body and patches of alopecia on the scalp. An oesophagogram taken one month before her admission showed a narrow area in the upper third of the oesophagus (Fig. 3) with some dilatation above it. A biopsy of one of the skin lesions

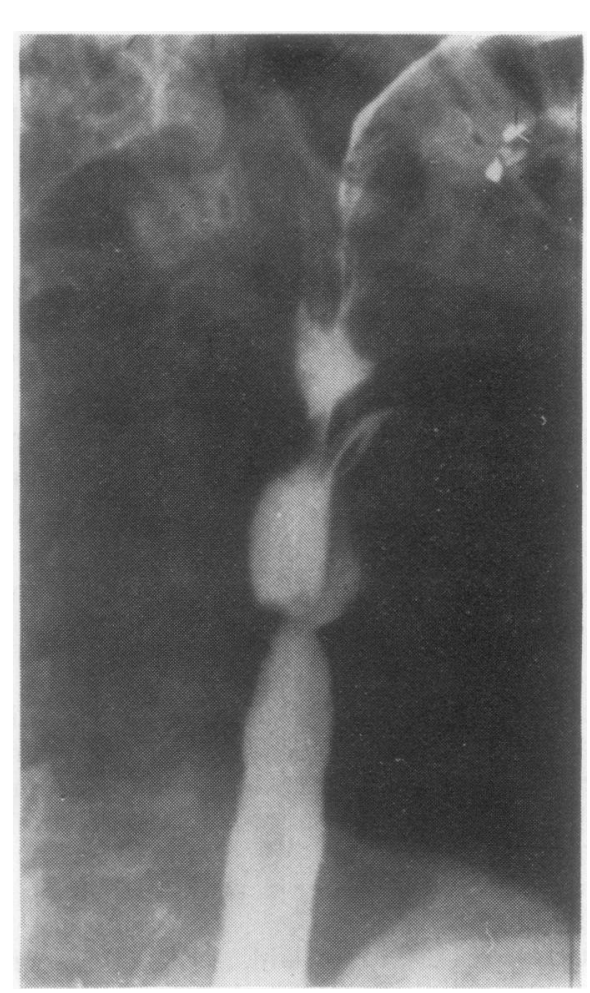

Fig. 2 Case 1. Postoperative barium swallow showing about $75 \%$ of full lumen at the site of anastomosis.

showed bullae situated deep in the epidermis, lymphocytic infiltration, and papillary swelling.

As in case 1 , the stricture, which was almost $1 \mathrm{~cm}$ in length and lay $3 \mathrm{~cm}$ below the pharyngooesophageal junction, was resected with subse- 3 quent end-to-end anastomosis. The orifice of the stricture was $2 \mathrm{~mm}$ in diameter, and on histological examination the mucosa, submucosa, and muscularis were found to be replaced by fibrous tissue. Since the operation the patient has been $\frac{7}{0}$ able to swallow normally. A barium swallow two years after the operation showed no evidence of $\mathcal{N}$ residual stricture (Fig. 4).

\section{Discussion}

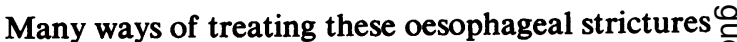

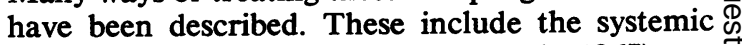
administration of steroids (Katz et al., 1967), repeated dilatation with bougies, colon transplant $\frac{0}{0}$ (Absolon et al., 1969), feeding gastrostomy, and $\frac{\mathrm{D}}{\mathrm{D}}$ restricting the patient to fluids and soft food.

Corticosteroids achieve only limited and tem- $\varrho$ porary benefit, and do not affect established stric-? tures. They were used in both our patients without 8 


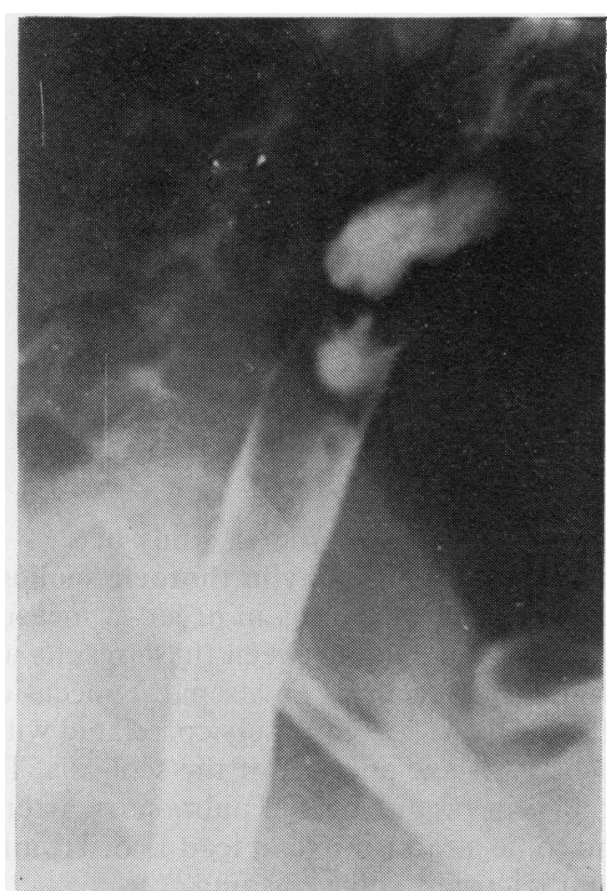

Fig. 3 Case 2. Preoperative barium swallow showing narrow stricture of the oesophagus.

effect. The passage of a bougie improves swallowing for a day or two only and may cause further bulla formation by trauma to the oesophageal mucosa. Because of changes in the patient's mouth, it may not be possible to pass bougies. A feeding gastrostomy is usually unacceptable to the patient. Unless there is extensive involvement of the oesophagus, which is uncommon, it seems preferable in these patients who are undernourished and debilitated to perform the lesser procedure of resection and end-to-end anastomosis rather than having recourse to a major operation such as a colon transplant.

From the results of surgery in our two patients, who are both swallowing well three years after operation, it seems that in the case of stenoses not exceeding $3 \mathrm{~cm}$ in length simple resection with end-to-end anastomosis is the best treatment for this type of stricture.

We thank Dr. Morteza Bagoghli for his help.

\section{References}

Absolon, K. B., Finney, L. A., Waddill, G. M., Jr., and Hatchett, C. (1969). Esophageal reconstruction -colon transplant-in two brothers with epidermolysis bullosa. Surgery, 65, 832-836.

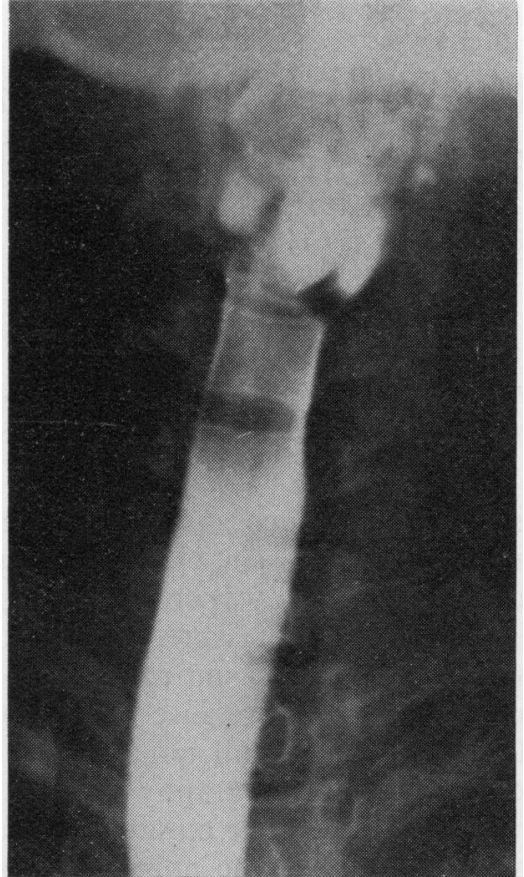

Fig. 4 Case 2. Barium swallow two years after operation with normal lumen at the site of anastomosis.

Bergenholtz, A., Olsson, O., Arwill, T., and Lunström, N. R. (1963). Die Epidermolysis bullosa hereditaria dystrophica mit Oesophagusveränderungen. Eine kritische Literatureübersicht mit Beschreibung von zwei Fällen. Archiv für klinische und experimentelle Dermatologie, 217, 518-533.

Dupree, E., Hodges, F., Jr., and Simon, J. L. (1969). Epidermolysis bullosa of the esophagus. American Journal of Diseases of Children, 117, 349-351.

Gedde-Dahl, T. (1971). Epidermolysis Bullosa, p. 81. Johns Hopkins Press, Baltimore.

Katz, J., Gryboski, J. D., Rosenbaum, H. M., and Spiro, H. M. (1967). Dysphagia in children with epidermolysis bullosa. Gastroenterology, 52, 259262.

Newcomer, V. D., Zeilenga, R. H., and Sternberg, T. H. (1956). Uncommon congenital abnormalities of the skin. Pediatric Clinics of North America, 3, 701-717.

Nix, T. E., and Christianson, H. B. (1965). Epidermolysis bullosa of the esophagus: report of two cases and review of the literature. Southern Medical Journal, 58, 612-620.

Schnyder, U. W., and Eichhoff, D. (1964). Zur Klinik und Genetik der dominant-dystrophischen Epidermolysis bullosa hereditaria. Archiv für klinische und experimentelle Dermatologie, 218, 62-90.

Requests for reprints to: Dr. Siavoush Sehhat, 160 Shah Avenue, Esfahan, Iran. 